\title{
EQUIVALENT METRICS GIVING DIFFERENT VALUES TO METRIC-DEPENDENT DIMENSION FUNCTIONS ${ }^{1}$
}

\author{
J. C. NICHOLS
}

In [1] K. Nagami and J. H. Roberts introduced metric-dependent dimension functions $d_{2}$ and $d_{3}$ defined on the class of all metric spaces. All definitions are given below. The definition of $d_{5}$ is due to R. E. Hodel [5]. The following relations hold for all metric spaces $(X, \rho)$ :

$$
d_{2}(X, \rho) \leqq d_{3}(X, \rho) \leqq d_{5}(X, \rho) \leqq \mu \operatorname{dim}(X, \rho) \leqq \operatorname{dim} X,
$$

where $\mu \mathrm{dim}$ is metric dimension as defined by Katětov [3], and $\operatorname{dim} X$ is covering dimension.

The following is a natural question. Suppose $d$ is $d_{2}, d_{3}, d_{5}$, or $\mu \mathrm{dim}$; and suppose $d(X, \rho)=r<n=\operatorname{dim} X$. Then for every $k(r \leqq k \leqq n)$ does there exist a topologically equivalent metric $\rho_{k}$ for $X$ such that $d\left(X, \rho_{k}\right)=k$ ? Roberts and Slaughter [2] answered this question in the affirmative when $d$ is $\mu \mathrm{dim}$. Roberts [6] answered this question in the affirmative for all separable metric spaces when $d$ is $d_{3}$. This paper answers this question in the affirmative for all metric spaces when $d$ is $d_{3}$ or $d_{5}$. The question remains unanswered when $d$ is $d_{2}$.

In the following if $S$ is a set, $|S|$ will denote the cardinality of $S$.

Definition. Let $\eta$ be any ordinal number. A metric space $(X, \rho)$ is said to have property $P(|\eta|, k, \rho)$ if given any collection of pairs of closed sets indexed by $\eta, \mathcal{e}=\left\{\left(C_{\alpha}, C_{\alpha}^{\prime}\right): \alpha<\eta\right\}$ such that there exists an $\epsilon>0$ with $\rho\left(C_{\alpha}, C_{\alpha}^{\prime}\right) \geqq \epsilon$ for all $\alpha<\eta$ then there exists a collection of closed sets $\left\{B_{\alpha}: \alpha<\eta\right\}$ such that $B_{\alpha}$ separates $X$ between $C_{\alpha}$ and $C_{\alpha}^{\prime}$ and order $\left\{B_{\alpha}: \alpha<\eta\right\} \leqq k$.

Definition. $d_{2}(X, \rho)$ is the smallest integer $n$ such that $(X, \rho)$ has property $P(n+1, n, \rho)$.

Definition. $d_{3}(X, \rho)$ is the smallest integer $n$ such that $(X, \rho)$ has property $P(m, n, \rho)$ for every integer $m$.

Definition. $d_{5}(X, \rho)$ is the smallest integer $n$ such that $(X, \rho)$ has property $P\left(\boldsymbol{\aleph}_{0}, n, \rho\right)$.

Definition. $\mu \operatorname{dim}(X, \rho)$ is the smallest integer $n$ such that for each $\epsilon>0$ there exists an open cover $\mathcal{U}$ of $X$ with

Presented to the Society, March 14, 1969 under the title Realization of a particular metric-dependent dimension function; received by the editors May 2, 1969.

1 This work is taken from the author's doctoral dissertation at Duke University. I would like to thank Dr. J. H. Roberts for his guidance in the preparation of this paper. 
(i) $\rho$-mesh $u \leqq \epsilon$ and

(ii) order $u \leqq n+1$.

Lemma. Suppose $(X, \rho)$ is a metric space, $f: X \rightarrow[0,1]$ is a continuous function and $\sigma(x, y)=\rho(x, y)+|f(x)-f(y)|$. Then $\sigma$ is a metric on $X$ topologically equivalent to $\rho$. (See $[4$, p. 199].)

Theorem 1. Suppose $(X, \rho)$ is a metric space, $f: X \rightarrow[0,1]$ a continuous function and $\sigma(x, y)=\rho(x, y)+|f(x)-f(y)|$.

(1) If $\eta$ is any ordinal number such that $\boldsymbol{\aleph}_{0} \leqq|\eta| \leqq 2 \aleph_{0}$ and $(X, \rho)$ has property $P(|\eta|, k, \rho)$ then $(X, \sigma)$ has property $P(|\eta|, k+1, \sigma)$.

(2) If $(X, \rho)$ has property $P(m, k, \rho)$ for every integer $m$ then $(X, \sigma)$ has property $P(m, k+1, \sigma)$ for every integer $m$.

Remark. To prove Theorem 2, the principle result of this paper, Theorem 1 is used only for the cases where $\eta$ is countable or finite.

Proof of Theorem 1. Let $\mathfrak{e}=\left\{\left(C_{\alpha}, C_{\alpha}^{\prime}\right): \alpha<\eta\right\}$ be any collection of pairs of closed sets with $|\eta| \geqq 2^{\aleph_{0}}$ and with $\sigma\left(C_{\alpha}, C_{\alpha}^{\prime}\right) \geqq \epsilon$ for all $\alpha<\eta$ and for some $\epsilon>0$. Choose an integer $N_{0}$ so that $1 / N_{0}<\epsilon / 4$. Since $|\eta| \leqq 2 \aleph_{0}$ there exists a set $T_{0}$ with $T_{0} \subseteq\left[0,1 / N_{0}\right)$ and with $\left|T_{0}\right|=|\eta|$. We can assume that $T_{0}=\left\{t_{\alpha}: \alpha<\eta\right\}$ where if $t_{\alpha}=t_{\beta}$ then $\alpha=\beta$. Let $S=\left\{0,1,2, \cdots, N_{0}\right\}$ and let $t_{\alpha}^{0}=0$ and $t_{\alpha}^{N_{0}+1}=1$ for all $\alpha<\eta$. For each $i, 1 \leqq i \leqq N_{0}$ and each $\alpha<\eta$ let $t_{\alpha}^{i}=t_{\alpha}+(i-1) / N_{0}$. Now for each $i \in S$ and each $\alpha<\eta$ define

$$
\begin{aligned}
& E_{\alpha}^{i}=\left\{x: t_{\alpha}^{i} \leqq f(x) \leqq t_{\alpha}^{i+1}\right\} \text { and define } \\
& D_{\alpha}^{i}=\left\{x: \rho\left(\left(C_{\alpha} \cap E_{\alpha}^{b}\right), x\right) \geqq \epsilon / 4\right\} .
\end{aligned}
$$

Then for $i \in S$ and $\alpha<\eta$ we have $\rho\left(C_{\alpha} \cap E_{\alpha}^{i}, C_{\alpha}^{\prime} \cap E_{\alpha}^{i}\right) \geqq 3 \epsilon / 4$ because for $x, y \in E_{\alpha}^{i}$ we have $|f(x)-f(y)| \leqq 1 / N_{0} \leqq \epsilon / 4$. Thus $D_{\alpha}^{i} \supseteq C_{\alpha}^{\prime} \cap E_{\alpha}^{i}$ for all $i \in S$ and $\alpha<\eta$. Now $D=\left\{\left(D_{\alpha}^{i}, C_{\alpha} \cap E_{\alpha}^{i}\right): i \in S, \alpha<\eta\right\}$ is a collection of pairs of closed sets with $\rho\left(D_{\alpha}^{i}, C_{\alpha} \cap E_{\alpha}^{i}\right) \geqq \epsilon / 4$. In Case (1) where $\aleph_{0} \leqq|\eta| \leqq 2 \aleph_{0}$, we have $|D|=|\eta|$. In Case (2) we have $|\eta|=m$ for some integer $m$ and $|D|=m N_{0}$. Thus in either case our hypothesis guarantees the existence of a collection of closed sets $\left\{B_{\alpha}^{i}: i \in S, \alpha<\eta\right\}$ with

(i) order $\left\{B_{\alpha}^{i}: i \in S, \alpha<\eta\right\} \leqq k$ and

(ii) $X-B_{\alpha}^{i}=U_{\alpha}^{i} \cup V_{\alpha}^{i}$ where $U_{\alpha}^{i}$ and $V_{\alpha}^{i}$ are disjoint open sets and $D_{\alpha}^{i} \subseteq V_{\alpha}^{i}$ and $C_{\alpha} \cap E_{\alpha}^{i} \subseteq U_{\alpha}^{i}$.

For each $\alpha<\eta$ we will modify the collection $\left\{B_{\alpha}^{i}: i \in S\right\}$ to obtain a closed set $B_{\alpha}$ separating $X$ between $C_{\alpha}$ and $C_{\alpha}^{\prime}$ using a variation of a method due to J. H. Roberts [7].

For each $i \in S$ and $\alpha<\eta$ define $L_{\alpha}^{i}=\left\{x: f(x)=t_{\alpha}^{i}\right\}$. Notice that 
$L_{\alpha}^{i}=E_{\alpha}^{i-1} \cap E_{\alpha}^{i}$, for $i$ such that $1 \leqq i \leqq N_{0}$. For each $\alpha<\eta$ let $L_{\alpha}^{N_{0+1}}=\varnothing$ and define

$$
\begin{aligned}
B_{\alpha} & =\bigcup_{j=0}^{N_{0}}\left[\left(B_{\alpha}^{j} \cap E_{\alpha}^{j}\right) \cup\left(L_{\alpha}^{j+1} \cap\left[\left(U_{\alpha}^{j}-U_{\alpha}^{j+1}\right) \cup\left(U_{\alpha}^{j+1}-U_{\alpha}^{j}\right)\right]\right)\right], \\
U_{\alpha} & =\bigcup_{j=0}^{N_{0}}\left(U_{\alpha}^{j} \cap E_{\alpha}^{j}\right)-B_{\alpha}, \\
V_{\alpha} & =\bigcup_{j=0}^{N_{0}}\left(V_{\alpha}^{j} \cap E_{\alpha}^{j}\right)-B_{\alpha} .
\end{aligned}
$$

Assertion 1. For each $\alpha<\eta B_{\alpha}$ is a closed set separating $X$ between $C_{\alpha}$ and $C_{\alpha}^{\prime}$.

Proof. First we show that $B_{\alpha}$ is closed. Let $H_{\alpha}=\cup_{j=0}^{N_{0}}\left(B_{\alpha}^{j} \cap E_{\alpha}^{j}\right)$ and let $G_{\alpha}=\bigcup_{j=0}^{N_{0}}\left(L_{\alpha}^{j+1} \cap\left[\left(U_{\alpha}^{j}-U_{\alpha}^{j+1}\right) \cup\left(U_{\alpha}^{j+1}-U_{\alpha}^{j}\right)\right]\right)$. It suffices to show that $\bar{G}_{\alpha} \subseteq B_{\alpha}$ since $H_{\alpha}$ is closed. If $x$ is a limit point of $G_{\alpha}$ then there exists some $k \in S$ such that $x$ is a limit point of $L_{\alpha}^{k+1} \cap\left[\left(U_{\alpha}^{k}-U_{\alpha}^{k+1}\right)\right.$ $\left.\cup\left(U_{\alpha}^{k+1}-U_{\alpha}^{k}\right)\right]$. We may assume then that $x$ is a limit point of $\left(U_{\alpha}^{k}-U_{\alpha}^{k+1}\right)$ hence a limit point of $U_{\alpha}^{k}$. But $X-B_{\alpha}^{k}=U_{\alpha}^{k} \cup V_{\alpha}^{k}$ where $U_{\alpha}^{k} \cap V_{\alpha}^{k}=\varnothing$. Thus either $x \in U_{\alpha}^{k}$ or $x \in B_{\alpha}^{k}$ and in either case $x \in B_{\alpha}$ so $B_{\alpha}$ is closed.

Next we show that $X-B_{\alpha}=U_{\alpha} \cup V_{\alpha}$. If $x \in X$, there exists $k \in S$ such that $x \in E_{\alpha}^{\boldsymbol{k}}$, since $\bigcup_{j=0}^{N_{0}} E_{\alpha}^{j}=X$. If $x \notin B_{\alpha}$ then surely $x \notin B_{\alpha}^{k} \cap E_{\alpha}^{\boldsymbol{k}}$. But $X-B_{\alpha}^{k}=U_{\alpha}^{k} \cup V_{\alpha}^{k}$ so $x$ is in one of $U_{\alpha}^{k}$ or $V_{\alpha}^{k}$ hence one of $U_{\alpha}$ or $V_{\alpha}$.

We show that $U_{\alpha} \cap V_{\alpha}=\varnothing$. If $x \in U_{\alpha}$ then either $x \in E_{\alpha}^{k}$ for exactly one $k \in S$ or $x \in\left(E_{\alpha}^{k} \cap E_{\alpha}^{k+1}\right)$ for exactly one $k \in S$. In the first case since $x \in U_{\alpha}^{k}$ we have $x \notin V_{\alpha}^{k}$ hence $x \notin V_{\alpha}$. In the second case we can suppose that $x \in U_{\alpha}^{k}$. The only possibility to have $x \in V_{\alpha}$ is to have $x \in V_{\alpha}^{k+1}$. But then $x \notin U_{\alpha}^{k+1}$ hence $x \in\left(U_{\alpha}^{k}-U_{\alpha}^{k+1}\right) \cap\left(E_{\alpha}^{k} \cap E_{\alpha}^{k+1}\right)$. Thus $x \in B_{\alpha}$ and $x \notin V_{\alpha}$ so we conclude that $U_{\alpha} \cap V_{\alpha}=\varnothing$.

To show that $C_{\alpha} \subseteq U_{\alpha}$ we first show that $C_{\alpha} \cap B_{\alpha}=\varnothing$. Let $x \in C_{\alpha}$ and suppose that $x \in E_{\alpha}^{k}$ for exactly one $k$. Then $x \notin L_{\alpha}^{j}$ for any $j \in S$. Now $\left(C_{\alpha} \cap E_{\alpha}^{k}\right) \cap B_{\alpha}^{\boldsymbol{k}}=\varnothing$ because $X-B_{\alpha}^{\boldsymbol{k}}=U_{\alpha}^{k} \cup V_{\alpha}^{\boldsymbol{k}}$ where $\left(C_{\alpha} \cap E_{\alpha}^{\boldsymbol{k}}\right)$ $\subseteq U_{\alpha}^{k}$. So in this case $x \notin B_{\alpha}$. If $x \in E_{\alpha}^{k} \cap E_{\alpha}^{k+1}$ for some $k \in S$ then $x \in U_{\alpha}^{k}$ and $x \in U_{\alpha}^{k+1}$. Thus $x \notin B_{\alpha}$ and $C_{\alpha} \cap B_{\alpha}=\varnothing$. Since $C_{\alpha}$ $\subseteq \bigcup_{j=0}^{N_{0}}\left(U_{\alpha}^{j} \cap E_{\alpha}^{j}\right)$ and $C_{\alpha} \cap B_{\alpha}=\varnothing$ we conclude that $C_{\alpha} \subseteq U_{\alpha}$.

From the definition of $D_{\alpha}^{j}$ it is clear that $C_{\alpha}^{\prime} \subseteq \bigcup_{j=0}^{N_{0}} D_{\alpha}^{j}$ but $D_{\alpha}^{j} \subseteq V_{\alpha}^{j}$ so $C_{\alpha}^{\prime} \subseteq \bigcup_{j=0}^{N_{0}} V_{\alpha}^{j}$. Thus if we show that $C_{\alpha}^{\prime} \cap B_{\alpha}=\varnothing$ we can conclude that $C_{\alpha}^{\prime} \subseteq V_{\alpha}$. Let $x \in C_{\alpha}^{\prime}$ and let $x \in E_{\alpha}^{k}$ for exactly one $k$. Then $x \in V_{\alpha}^{k}$ so $x \notin B_{\alpha}^{k}$ hence $x \notin B_{\alpha}$. If $x$ is in $E_{\alpha}^{k} \cap E_{\alpha}^{k+1}$ then $x \in V_{\alpha}^{k}$ and $x \in V_{\alpha}^{k+1}$ hence $x \notin U_{\alpha}^{\boldsymbol{k}}$ and $x \notin U_{\alpha}^{k+1}$ so $x \notin B_{\alpha}$. Thus $C_{\alpha}^{\prime} \subseteq V_{\alpha}$. 
We show that $U_{\alpha}$ is open. Let $x \in U_{\alpha}$. Then $x \notin B_{\alpha}$ a closed set so there exists an open set $M_{x}$ containing $x$ with $M_{x} \cap B_{\alpha}=\varnothing$. Suppose $x \in E_{\alpha}^{k}$ for some unique $k$. Then $x$ is in the interior of $E_{\alpha}^{k}$ so there exists an open set $N_{x}$ with $x \in N_{x} \subseteq E_{\alpha}^{k}$. Then $x \in M_{x} \cap N_{x} \cap U_{\alpha}^{k} \subseteq U_{\alpha}$. Suppose $x \in L_{\alpha}^{k+1}$ some $k \in S$. Since $x$ is in the interior of $E_{\alpha}^{k} \cup E_{\alpha}^{k+1}$ choose an open set $N_{x}$ so that $x \in N_{x} \subseteq E_{\alpha}^{k} \cup E_{\alpha}^{k+1}$. Since $x \in L_{\alpha}^{k+1}$ and $x \in U_{\alpha}^{k}$ and $x \notin B_{\alpha} \quad$ we have $x \in U_{\alpha}^{k+1}$. Thus $x \in\left(U_{\alpha}^{k} \cap U_{\alpha}^{k+1}\right) \cap M_{x} \cap N_{x}$ $\subseteq U_{\alpha}$. Thus $U_{\alpha}$ is open. A similar argument shows that $V_{\alpha}$ is open. This completes the proof of Assertion 1.

Assertion 2. Order $\left\{B_{\alpha}: \alpha<\eta\right\} \leqq k+1$.

Proof. Let $P_{\alpha}=\cup_{j=0}^{N_{0}}\left(B_{\alpha}^{j} \cap E_{\alpha}^{j}\right)$. Then order $\left\{P_{\alpha}: \alpha<\eta\right\} \leqq k$ since order $\left\{B_{\alpha}^{j}: j \in S, \alpha<\eta\right\} \leqq k$. For $\alpha<\eta$ let $Q_{\alpha}=\bigcup_{j=1}^{N_{0}} L_{\alpha}^{j}$. Now order $\left\{Q_{\alpha}: \alpha<\eta\right\} \leqq 1$. Hence order $\left\{B_{\alpha}: \alpha<\eta\right\} \leqq$ order $\left\{\left(P_{\alpha} \cap Q_{\alpha}\right): \alpha<\eta\right\}$ $\leqq k+1$. This completes the proof of the theorem.

Corollary. Let $(X, \rho)$ be a metric space, $f: X \rightarrow[0,1]$ a continuous function, $\sigma(x, y)=\rho(x, y)+|f(x)-f(y)|$, and let $d$ be $d_{3}$ or $d_{5}$. If $d(X, \rho)$ $\leqq k$ then $k \leqq d(X, \sigma) \leqq k+1$.

THEOREM 2. Let $(X, \rho)$ be a metric space and let $d$ be $d_{3}$ or $d_{5}$. Suppose $d(X, \rho)=r<n=\operatorname{dim} X$. Then for each $k, r \leqq k \leqq n$ there exists a topologically equivalent metric $\rho_{k}$ for $X$ such that $d\left(X, \rho_{k}\right)=k$.

Proof. Let $C_{1}, C_{1}^{\prime} ; C_{2}, C_{2}^{\prime} ; \cdots, C_{n}, C_{n}^{\prime}$ be $n$ pairs of disjoint closed sets with the property that if for each $i=1, \cdots, n B_{i}$ is a closed set separating $C_{i}$ and $C_{i}^{\prime}$, then $\bigcap_{i=1}^{n} B_{i} \neq \varnothing$. This is possible since $\operatorname{dim} X$ $=n$. For each $i=1, \cdots, n$ let $f_{i}: X \rightarrow[0,1]$ such that $f$ is continuous, $f_{i}\left(C_{i}\right)=0$ and $f_{i}\left(C_{i}^{\prime}\right)=1$. For each $i=1, \cdots, n$ define $\sigma_{i}: X \times X$ $\rightarrow$ real numbers by

$$
\sigma_{i}(x, y)=\rho(x, y)+\sum_{j=1}^{i}\left|f_{j}(x)-f_{j}(y)\right| .
$$

Now $\sigma_{n}\left(C_{i}, C_{i}^{\prime}\right) \geqq 1$ for all $i=1, \cdots, n$ thus $d\left(X, \sigma_{n}\right) \geqq n$. But by the above corollary $d\left(X, \sigma_{i+1}\right) \leqq d\left(X, \sigma_{i}\right)+1$. Thus all values $k, r \leqq k \leqq n$, are assumed and the theorem is proved.

\section{REFERENCES}

1. Keio Nagami and J. H. Roberts, $A$ study of metric-dependent dimension functions, Trans. Amer. Math. Soc. 129 (1967), 414-435.

2. J. H. Roberts and F. G. Slaughter, Jr., Metric dimension and equivalent metrics, Fund. Math. 62 (1968), 1-5.

3. M. Katětov, On the relations between the metric and topological dimensions, Czechoslovak Math. J. 8 (1958), 163-166. 
4. Witold Hurewicz, Über Einbettung separable Räume in gleich dimensionale kompakte Räume, Monatshef te für Math. und Physik 37 (1930), 199-208.

5. R. E. Hodel, Note on metric-dependent dimension functions, Fund. Math. 61 (1967), 83-89.

6. J. H. Roberts, Realizability of metric-dependent dimensions, Proc. Amer. Math Soc. 19 (1968), 1439-1442.

7. - Dimension function $d_{2}$ and the covering dimension, Duke Math. J. (to appear).

DUKE UnIVERSITY 